\section{Vergütung gestalten}

\author{
Eckhard Eyer
}

Der neue Tarifvertrag für den öffentlichen Dienst erlaubt variable Leistungsentgelte. Jede Organisation kann nun auf ihre Verhältnisse zugeschnittene Vergütungsverfahren entwickeln. Zur schrittweisen Gestaltung und Einführung eines Leistungsentgeltsystems empfiehlt sich der Aufbau eines systematischen Projektmanagements.

Der neue Tarifvertrag für den öffentlichen Dienst (TVöD) bringt eine grundlegende Änderung der Vergütung auch in der Sozialwirtschaft mit sich. Bislang orientierte sich die Bezahlung im öffentlichen Dienst stark am Senioritäts- und Alimentationsprinzip und erfolgte nach Qualifikation, Lebensalter, Familienstand und Kinderzahl. Künftig wird das Monatsgehalt aus einem Grundentgelt, das mit zunehmender Berufserfahrung in festgelegten Zeitabständen erhöht wird, und einem variablen Leistungsentgelt bestehen. Das Leistungsentgelt soll der Verbesserung der öffentlichen Dienstleistungen sowie der Stärkung der Motivation, Eigenverantwortung und Führungskompetenz der Mitarbeiter dienen.

Bei der Gestaltung des Leistungsentgelts lassen die Tarifvertragsparteien den Einrichtungen und Betrieben großen Spielraum. Während der Bund im wesentlichen nur die Höhe des Leistungsentgelts mit anfangs einem Prozent der ständigen Monatsentgelte des Vorjahres aller unter den Geltungsbereich des TVöD fallenden Beschäftigten des jeweiligen Arbeitgebers regelt und als Zielgröße für die Höhe acht Prozent angibt, treffen die Kommunen darüber hinaus weitere Festlegungen.

Formen des Leistungsentgelts sind danach:

- Leistungsprämie aufgrund von Zielvereinbarungen
- Leistungszulage aufgrund systematischer Leistungsbewertung

- Erfolgsprämie aufgrund des wirtschaftlichen Erfolgs des Unternehmens

Die inhaltliche Ausgestaltung der Leistungsentgeltformen wird den Betriebsparteien überlassen, die dazu eine Dienst- oder Betriebsvereinbarung schließen müssen.

Die Erfolgsprämie, die sich am Erfolg der Einrichtung orientiert, beruht auf der Messung von Kennzahlen, der Bilanz oder Gewinn- und Verlustrechnung. Erfolgsprämien können tariflich nur zusätzlich zum Leistungsentgelt gezahlt werden und nicht an die Stelle des Leistungsentgeltes treten (§ 18 Abs. 4 TVöD).

Eine Kombination der Leistungsentgeltmethoden ist möglich. So kann es sinnvoll sein, eine Teamleistungsprämie aufgrund der Zielvereinbarung und der Zielerreichung eines Teams mit einer systematischen Leistungsbewertung zu kombinieren, die die individuelle Leistung der Teammitglieder honoriert (vgl. Grafik). Durch die Kombination der Leistungsentgeltmethoden erhöht sich das Leistungsentgelt nicht.

\section{Was Personalmanagement und Mitarbeitervertreter neu heraus- fordert}

Der neue TVöD ist, wie die Erfahrung in einer Reihe von Unternehmen der Sozialwirtschaft bereits zeigt, eine große Herausforderung für die betrieblichen Akteure, denn was Vergütungsfragen betrifft, wurden Personalabteilung und Betriebsrat in der Sozialwirtschaft bislang nicht gestaltend, sondern lediglich administrativ und kontrollierend gefordert.

Die Betriebsparteien - also Personalleitung und Betriebsrat oder Mit-

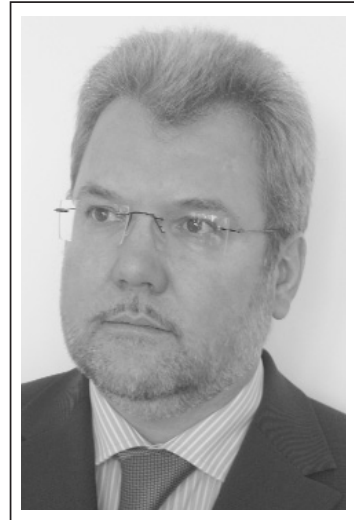

Eckhard Eyer ist Diplomingenieur, Diplomkaufmann und Vergütungsberater.

Er ist geschäfts-

führender Gesellschafter der Perspektive Eyer Consulting in Köln. Eyer beriet das erste »Modellprojekt Leistungsentgelt « der Caritas, er ist Berater der »Projektgesellschaft innovatives Arbeiten in caritativen Unternehmen « und hat bei verschiedenen Wohlfahrtsverbänden in der ambulanten und stationären Altenpflege sowie der Jugend- und Behindertenarbeit, Leistungsund Erfolgsvergütung eingeführt. Zunehmend unterstützt er Unternehmen der Sozialwirtschaft die betriebliche Entgeltsysteme statt des TVöD einführen. Er ist Herausgeber des Buches »Entgeltsysteme für Dienstleister « und Autor des Buches »Leistungsentgelt nach TVöD erfolgreich einführen «. Zudem ist er Lehrbeauftragter an der Universität Trier.

Internet http://www.eyer.de 


\section{Wie man Leistungsentgeltsysteme einführt}

Zur schrittweisen Gestaltung und Einführung eines Leistungsentgeltsystems empfiehlt sich der Aufbau eines systematischen Projektmanagements. Wesentliches Kennzeichen eines Projektmanagements ist die arbeitsteilige Ausführung von vorgegebenen Aufgaben, die durch ein Steuerungsgremium inhaltlich und zeitlich koordiniert und organisiert werden.

- Aufbau der Projektstruktur Um ein Leistungsentgelt, das auf die betrieblichen Bedürfnisse zugeschnitten ist, zu finden und einzuführen, ist die Beteiligung von Betriebs- oder Personalrat und Arbeitgebervertretung notwendig. Hierfür hat der TVöD eine »Betriebliche Kommission « vorgesehen, deren Mitglieder jeweils zur Hälfte von beiden Betriebsparteien benannt werden. Ihre Aufgabe ist die Mitwirkung bei der Entwicklung und dem Controlling des Leistungsentgeltsystems sowie die Beratung von Beschwerden. Diese Kommission sollte die Funktion der Steuerungsgruppe übernehmen, die sich - falls erforderlich - durch interne Fachleute oder externe Berater verstärken kann. Die Steuerungsgruppe setzt konkrete Ziele, plant die Arbeitsaufgaben im Zeitablauf und delegiert diese Aufgaben erforderlichenfalls an untergeordnete Projektteams. Die Zahl der Projektteams ist abhängig vom Umfang der anstehenden Aufgaben. So ist zum Beispiel entscheidend,

- ob in einer Einrichtung nur ein einheitliches Leistungsentgeltsystem eingeführt werden soll oder mehrere Systeme parallel und

- ob in allen Bereichen einer Einrichtung zeitgleich ein Leistungsentgeltsystem eingeführt wird oder nur in einem Teilbereich quasi als Pilotprojekt - begonnen wird.

Auch ist zu klären,

- ob in einem Projektteam ein Leistungsentgeltsystem, beispielsweise ein Verfahren zur systematischen Leistungsbewertung, von der Gestaltung über die Schulung der Beurteiler bis hin zur Einführung erarbeitet wird oder

- ob es Projektteams fachlich differenziert zur Systemgestaltung, zur

\section{Vier Tipps für die Praxis}

Die Erfahrungen des Autors in verschiedenen Projekten zum leistungsund erfolgsabhängigen Entgelt in der Sozialwirtschaft lassen ihn zu vier Tipps für die Praxis kommen.

1.

Das Leistungsentgelt ist eine Chance für die Verbesserung der Per- sonalführung und Motivation der Mitarbeiter in den Einrichtungen.

2 Die Gestaltung und Einführung des Leistungsentgeltes ist eine

- große Herausforderung für alle Beteiligte; sie sollte nicht unterschätzt werden: Der Prozess braucht ausreichend Zeit.

2 Die frühzeitige und ausführliche Information und Kommunikati-

3. on mit den Mitarbeitern ist ein wichtiger Aspekt der Einführung; damit werden Ängste abgebaut und Chancen aufgezeigt.

Kennzahlen und Audits für die Leistungsvergütung sollten mit dem Qualitätsmanagement synchronisiert werden.

Eckhard Eyer

Information, zur Qualifizierung u. ä. geben soll.

Der Projektleiter koordiniert die Arbeiten innerhalb seines Teams, kommuniziert mit den Projektleitern paralleler Projektteams und berichtet über die Arbeitsergebnisse in der Steuerungsgruppe. Zur Mitarbeit in den Projektteams werden zum einen Mitarbeiter der Personalabteilung, die mit Vergütungsfragen betraut sind, herangezogen sowie auch Mitarbeiter und Führungskräfte der Bereiche, in denen ein Leistungsentgelt eingeführt werden soll.

\section{- Projektplanung}

Sobald aufgrund einer Grobplanung die Strukturen für das Projekt aufgebaut worden sind, erfolgt die detaillierte Planung der Projektschritte im Zeitablauf. Für die Einführung eines Leistungsentgeltsystems sind im Wesentlichen folgende Schritte festzulegen:

- Entscheidung für die Form des Leistungsentgeltsystems

- Festlegung des Geltungsbereichs

- Auswahl und erforderlichenfalls ergänzende Qualifizierung der Projektteammitglieder

- Erarbeitung des Leistungsentgeltsystems

- Information der Mitarbeiter (mündlich, schriftlich)

- Schulung der Mitarbeiter (Leistungsbewertung, Zielvereinbarung, Führung von Mitarbeitergesprächen)

- gegebenenfalls Durchführung eines »Trockenlaufs«, also die Er-

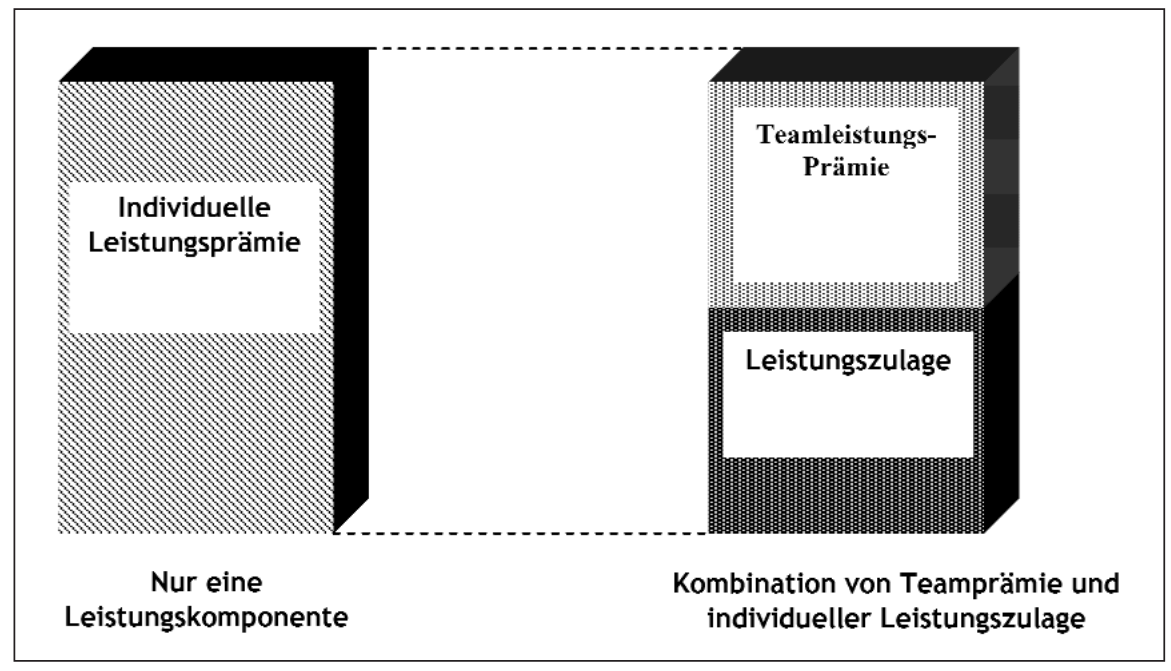


probung des Leistungsentgeltsystems ohne Entgeltrelevanz

- verwaltungstechnische Umsetzung der Leistungsvergütung (Anbindung an Gehaltsabrechnung)

- Abschätzung des Gesamtaufwandes und Bereitstellung zusätzlicher Ressourcen

Die ergänzende Festlegung von Meilensteinen, also Zeitpunkten, zu denen bestimmte Aufgaben abgeschlossen sein sollten, ist hilfreich, um Teilziele für die Mitwirkenden zu setzen und den Projektablauf zu steuern.

- Projektdurchführung und Projektabschluss

Nach der Planung werden die Aufgabenpakete den Projektmitwirkenden zugeordnet und von ihnen ausgeführt. In Zeitabständen erfolgt eine Rückmeldung aus den Projektteams über den Projektleiter an die Steuerungsgruppe, die den Projektverlauf auf diese Weise begleitet und bei Störungen eingreift.

Zum Abschluss des Projektes ist eine ausführliche Bewertung des Pro- jektes aus Sicht der Steuerungsgruppe wie auch der in den Projektteams einbezogenen Mitarbeiter sinnvoll. Die Erfahrungen aus der Projektarbeit sollten auf diese Weise zusammengestellt und für zukünftige Arbeiten genutzt werden.

\section{Literatur}

Eyer, E.: Leistungsentgelte in Non-ProfitUnternehmen - eine Paradoxie? In: Personal, 1/2003, S. 34-37.

Eyer, E.; Haussmann, T.: Zielvereinbarung und variable Vergütung. Ein Leitfaden nicht nur für Führungskräfte. Wiesbaden: Gabler, 3. erw. Auflage 2005.

Eyer, E.; Haussmann, T.: Leistungsentgelt nach TVöD erfolgreich einführen. Wiesbaden, Gabler Verlag, 2006.

Eyer, E. (Hg.): Entgeltsysteme für Dienstleister: Grundvergütung - Zielvereinbarung - Erfolgsbeteiligung. Düsseldorf. Symposion 2004,

\section{Literaturtipp}

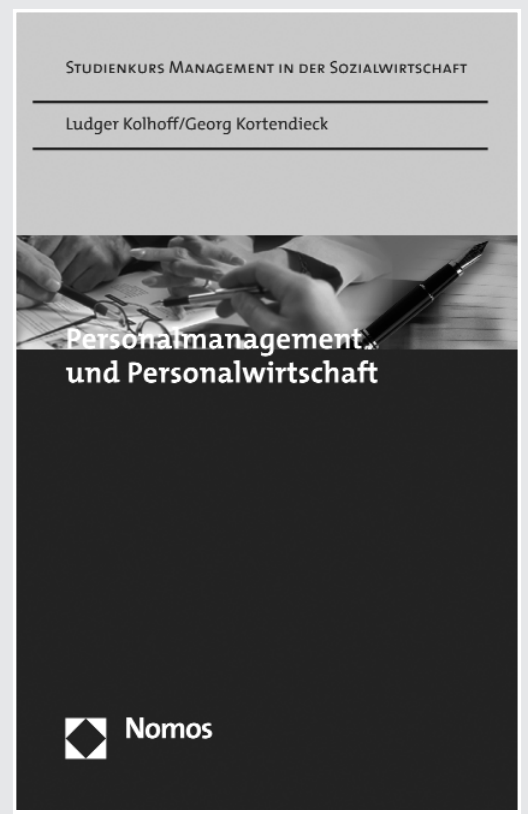

In einem Band aus der Reihe »Studienkurs Management in der Sozialwirtschaft « erhalten Führungskräfte einen kompakten Überblick über die wichtigsten Felder des Personalmanagements und der Personalwirtschaft. Beleuchtet werden die grundlegenden Hintergründe und zentrale Aspekte der Personalführung, Personalplanung, Personalbeschafffung, Personalhonorierung, Personalbeurteilung und auch der Personalentwicklung in der Sozialwirtschaft.

Ludger Kolhoff/Georg Kortendieck: Personalmanagement und Personalwirtschaft. Nomos Verlagsgesellschaft, Baden-Baden 2006. 176 Seiten. 19,80 Euro. ISBN 3-8329-1633-4. 